\section{The Impact of Somatic and Germline Mutations in Myelodysplastic Syndromes and Related Disorders}

\author{
Rafael Bejar, MD, PhD, and Peter L. Greenberg, MD
}

Molecular profiling has identified critical genetic derangements contributing to the clinical phenotypes of patients with myelodysplastic syndromes (MDS) and related disorders. Molecular insights have demonstrated how specific somatic gene mutations contribute to the development and clinical outcomes of MDS, including their propensity to progress to more aggressive stages, such as acute myeloid leukemia (AML). Despite these findings, ambiguity remains about how best to use somatic mutations to determine prognosis and aid in diagnosis. Meanwhile, several germline mutations predisposing to myeloid malignancies have been identified that will change how we perform genetic testing for MDS and interpret the results. This commentary focuses on these issues.

\section{Prognostic Use of Somatic Mutations in MDS}

Much has been learned about the genetic basis of MDS by identifying somatic mutations of recurrently altered genes in the vast majority of patients. Sequencing 40 genes will identify one or more pathogenic mutations in more than $90 \%$ of patients with MDS. ${ }^{1,2}$ These genes belong to a variety of molecular pathways, including RNA splicing, epigenetic regulation, hematopoietic transcription factors, and growth factor signaling. However, only a handful are mutated in more than $10 \%$ of patients (Table 1). ${ }^{3}$ Increasingly, somatic mutation testing is being performed with large panels of at least 40 genes with high sensitivity to detect even small subclones. Data have indicated that the more mutations noted, the worse the prognosis, although certain mutations are clearly more adverse than others. ${ }^{1}$

Approximately 15 genes have been shown to have negative prognostic import, whereas only one mutated gene (SF3B1) has a favorable association with patient outcomes (Table 2). Recent studies have shown that several of these mutations are prognostically additive to the clinical risk-based systems, such as the International Prognostic Scoring System (IPSS) and IPSS-R (revised), ${ }^{1,2,4-6}$ including for those patients undergoing allogeneic stem cell transplantation. ${ }^{7,8}$

In particular, mutations in 5 genes (TP53, EZH2, ETV6, RUNX1, ASXL1) have been shown to have independent adverse prognostic significance in several studies. Of these, TP53 mutations are consistently the most adverse. They are found in roughly $50 \%$ of patients with MDS with a complex karyotype, yet are associated with an even shorter overall survival than the combination of karyotype and clinical features would predict. In contrast, SF3B1 mutations, which are strongly associated with ring sideroblasts, indicate a better than predicted disease risk. ${ }^{9}$

\begin{tabular}{|c|c|}
\hline$>20 \%$ & $T E T 2$, a SF3B1, ASXL1"a \\
\hline $10 \%-20 \%$ & DNMT3A, RUNX1, SRSF2a \\
\hline $5 \%-10 \%$ & TP53, EZH2, ${ }^{a} N R A S,{ }^{a}$ U2AF1, ZRSR2 \\
\hline$<5 \%$ & $C B L$, a SETBP 1, a IDH1/2, ETV6, JAK2 \\
\hline
\end{tabular}

Data from Greenberg PL, Stone RM, Al Kali A, et al. NCCN Clinical Practice Guidelines in Oncology: Myelodysplastic Syndromes, Version 2.2017. Accessed October 25, 2016. To view the most recent version of thes guidelines, visit NCCN.org.

${ }^{a}$ More frequent in chronic myelomonocytic leukemia.

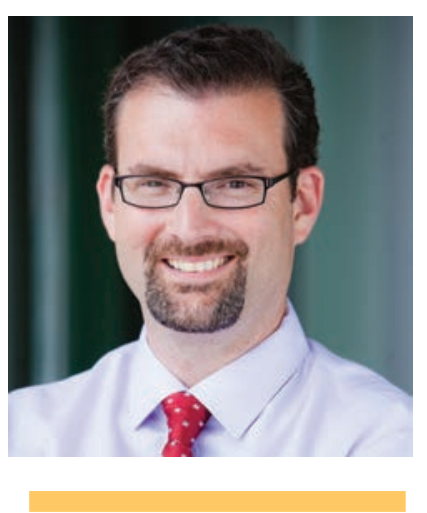

Rafael Bejar, MD, PhD

Rafael Bejar, MD, PhD, is an Assistant Professor and physicianscientist at UC San Diego Moores Cancer Center where he has served on the faculty since 2012. His clinical practice is focused on the care of patients with MDS and related disorders. He has established an MDS Center of Excellence recognized by the MDS Foundation that offers expertise in hematopathology, clinical trials, genetic testing, and access to allogeneic stem cell transplantation. In addition, he teaches hematology, oncology, and cancer science courses at the medical school and in the biomedical sciences graduate program. Dr. Bejar's laboratory research is focused on the molecular basis of myeloid disorders and the use of genetics to inform the care of patients with MDS. He has collaborated with colleagues around the globe to define how acquired mutations in MDS are associated with clinical features and how they might predict outcomes and refine the prediction of prognosis for patients. He is a member of the International Working Group for Prognosis in MDS (IWG-PM) molecular subcommittee, which is now working to incorporate molecular mutations into the standard risk evaluation for MDS.

The ideas and viewpoints expressed in this commentary are those of the author and do not necessarily represent any policy, position, or program of NCCN 
Bejar and Greenberg

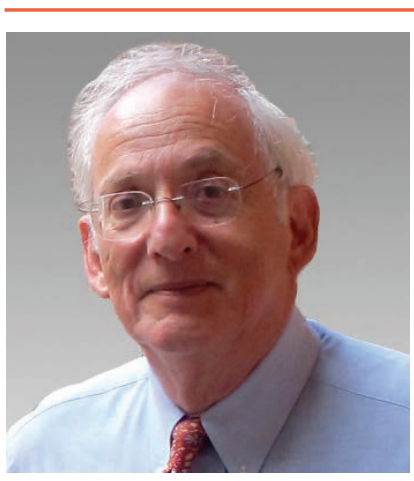

Peter L. Greenberg, MD

Peter L. Greenberg, MD, is Professor of Medicine (Hematology), Emeritus, at Stanford Cancer Institute. Dr Greenberg's laboratory research focuses on evaluating molecular abnormalities in myelodysplastic syndromes (MDS), with specific interest in gene expression profiling of marrow stem and progenitor cells using RNA sequencing and microarray methodologies and proteomic analysis of aberrant antigen expression in plasma. As Director of the Stanford MDS Center, his clinical research involves design and coordination of clinical trials using experimental drugs with biologic focus for patients with lower- and higher-risk MDS not responding to standard therapies. He is Coordinator of the International Working Group for Prognosis in MDS (IWG-PM), which generated the revised MDS classification system (the IPSS-R) and is now evaluating the impact of molecular mutations on this risk-based prognostic system. He is Chair of the NCCN MDS Panel.
Consensus guidelines on how to incorporate prognostically significant mutations in the care of patients with MDS are being developed. In the meantime, they may be used to refine the prognosis in patients predicted to have specified risk by established scoring systems.

\section{Diagnostic Use of Somatic Mutations}

MDS can be difficult to distinguish from more benign conditions capable of causing clinically significant cytopenias. Existing criteria for MDS are designed to identify individuals who clearly have the disorder, but may fail to provide a diagnosis for patients with unexplained cytopenias that fall short of these requirements. ${ }^{10}$ The features central for the diagnosis of MDS include well-defined dysplasia in one or more hematopoietic cell lines in addition to cytopenias. Cytopenias need to be persistent (usually over months) and not be due to other underlying conditions. ${ }^{11}$ Further, analysis of studies-including the MDS databases that generated the IPSS and IPSS-R - have shown that use of standard hematologic values to define cytopenic cut points for MDS diagnosis are more appropriate than the WHOrecommended prognostic cytopenia cut points. ${ }^{12}$ The WHO guidelines recognize that morphologic criteria alone may not be sufficient to diagnose every case of MDS. Thus they include a table of clonal chromosomal abnormalities that can serve as presumptive evidence for the disease in cases without gross dysplasia or increased blast proportions. ${ }^{11}$ Even though they represent evidence of clonal hematopoiesis, somatic mutations are not considered diagnostic of MDS, with one exception: in the recent revision of the WHO classification, the presence of a typical somatic SF3B1 mutation in patients with cytopenia and at least $5 \%$ ring sideroblasts is considered diagnostic of MDS.

Uncertainty remains regarding how to interpret somatic mutations in other genes in patients with cytopenia who do not meet existing diagnostic criteria for MDS. The basis for this ambiguity relates to the finding that somatic mutations in myeloid malignancy genes like DNMT3A and TET2 are remarkably common in healthy individuals with normal hematopoietic parameters. ${ }^{13,14}$

The incidence of these mutations increases greatly with age, approaching 15\% for persons in their 70s, which is the typical age of diagnosis for patients with MDS. The incidence of MDS is nearly 100 -fold lower, however, indicating that progression from clonal hematopoiesis to MDS or another hematologic malignancy is actually rare, estimated to be about $1 \%$ per year. This is comparable to the rate of progression from monoclonal gammopathy of undetermined significance to multiple myeloma. Therefore, identification of a blood-borne somatic mutation in a person without an identified hematologic disorder has been termed clonal hematopoiesis of indeterminate potential (CHIP) and is not diagnostic of MDS..$^{15,16}$

However, certain features of mutations may make them more likely to be related to a person's cytopenias. Mutations of DNMT3A and TET2 are common in CHIP,

\begin{tabular}{|c|c|}
\hline Splicesome genes & U2AF1, SRSF2, ZRSR2, SF3B1 ${ }^{a}$ \\
\hline Epigenetic modifiers & TET2, DNMT3A, EZH2, ASLX1, IDH1/2 \\
\hline Transcription factors & RUNX1, ETV6, WT1, GATA2 \\
\hline Activated signaling & NF1, NRAS, CBL, PTPN11, JAK2 \\
\hline Tumor suppressor & TP53 \\
\hline Cohesin factors & STAG2, SMC3 \\
\hline
\end{tabular}

${ }^{\mathrm{a} G o o d}$ prognosis, associated with ring sideroblasts. 
but mutated genes are more rare (SF3B1, RUNX1, EZH2). Most people with CHIP harbor a single gene mutation found in less than $25 \%$ of nucleated blood cells. In contrast, patients with MDS typically have 2 or more commonly mutated genes representing the bulk of all blood or bone marrow cells. The abundance of mutations appears to be an important risk factor for CHIP, because those with more than $25 \%$ clonal involvement (ie, approximately $12 \%$ variant allele frequency [VAF]) have the greatest risk of progression to malignancy. ${ }^{13}$

In patients with unexplained or idiopathic cytopenia of unknown significance (ICUS), the rates of somatic mutation are much higher than they are in an agematched general population, at nearly $40 \%$ (vs $15 \%$ for CHIP). ${ }^{17}$ These patients are said to have clonal cytopenia of unknown significance (CCUS), which may be an important risk factor for disease progression, because the average clonal burden in these patients is comparable to that in MDS. In a recent study of patients with cytopenia who experience progression to MDS or AML after a prior nondiagnostic biopsy, more than $90 \%$ were noted to have CCUS on the initial study. ${ }^{18}$ The mean number of mutated genes was 2 (vs 1 for CHIP), and the average clonal burden was high ( $>80 \%$ of cells or VAF $>40 \%$ ). However, not all patients with CCUS will experience progression to a malignancy in short order. ${ }^{19}$

\section{Germline Mutations in MDS}

The genetic bases of several distinct hereditary syndromes characterized by familial predisposition to myeloid neoplasms have recently been elucidated. Germline mutations in more than 20 genes have been linked to these disorders, including several that are somatically mutated in nonfamilial cases of disease. Several of these mutated genes are associated with partial penetrance and a long latency to transformation, such as DDX41, which can cause disease even after the fifth decade of life. ${ }^{20,21}$ Germline variants in genes such as TERT, TERC, DKC1, and GATA2 can be present in the absence of overt features commonly associated with them in typical/ classic syndromic cases. Germline variants can also arise as spontaneous mutations in persons with no family history (Table 3).,22-24 Younger patients with MDS and those with therapy-related myeloid malignancies may be more likely to harbor germline variants in these predisposition genes. Germline variants should be considered in patients when mutations in these genes are seen in tumor sequencing tests, especially when the mutations are biallelic or consistent with their presence in the germline (ie, with a VAF 50\%).

Identification of germline predisposition mutations has important clinical ramifications. First, it may identify family members at risk, many of whom will have clonal hematopoiesis before developing disease. Second, it allows for screening of potential related bone marrow stem cell donors who may likewise be affected. Whenever possible, confirmatory genetic testing should be performed on constitutional tissue, preferably on skin fibroblasts, to exclude somatic mutations

\begin{tabular}{|l|l|}
\hline $\begin{array}{l}\text { Table 3. Germline Mutation With Predisposition to } \\
\text { Develop Myeloid Clonal Neoplasms }\end{array}$ \\
\hline Familial MDS/AML & $\begin{array}{l}\text { RUNX1, GATA2, ETV6, CEBPA, } \\
\text { DDX41, ANKRD26, SRP72 }\end{array}$ \\
\hline $\begin{array}{l}\text { Classical inherited BMF } \\
\text { syndromes }\end{array}$ & TERTITERC, FANC, DKC1, ELANE, \\
\hline Other inherited syndromes & TP53, PTPN11, CBL, KRAS, NF1, \\
& BLM, ATG2B, GSKIP, BRCA1/2 \\
\hline
\end{tabular}


Bejar and Greenberg

\begin{tabular}{|l}
\hline Table 4. Recommendations for Gene Mutation Testing \\
Diagnostic adjunct in patients with suspected MDS \\
- Broad gene myeloid somatic mutation panel \\
• Clinical follow-up every 6 months for patients with CCUS \\
Prognostic adjunct in patients with MDS \\
- Targeted somatic mutation panel including SF3B1, TP53, ASXL1, RUNX1,EZH2, \\
ETV6 \\
For patients with MDS with relevant family history, young age, or syndromic features \\
\hline Germline gene predisposition panel testing
\end{tabular}

Abbreviations: CCUS, clonal hematopoiesis of undetermined significance, MDS, myelodysplastic syndromes.

and avoid false-negatives due to peripheral blood somatic mosaicism. Clinicians can access GeneTests, a medical genetics information resource from Bio-Reference Laboratories (available at www.genetests.org) to find laboratories to perform CLIA (Clinical Laboratory Improvement Amendments)-approved sequencing.

\section{Conclusions}

Genetic testing with large gene panels has become increasingly common for patients with MDS. Recommendations for such testing are indicated in Table 4. Somatic mutations can carry independent prognostic information and, in the case of patients with SF3B1 mutations and ring sideroblasts, may aid in diagnosis. However, somatic mutations indicative of clonal hematopoiesis occur with such frequency in healthy individuals that their presence alone in a patient with cytopenia cannot be used as presumptive evidence of MDS or evidence against alternative diagnoses. Genetic testing to identify patients with CCUS may be useful because these individuals are likely at the greatest risk of disease progression.

An absence of mutations after testing with a broad gene panel may have negative predictive value, potentially indicating an alternative diagnosis or a low risk of disease progression. Testing for germline predisposition mutations should be considered in patients with a provocative family history, younger age at diagnosis, or associated syndromic findings. Because germline variants may be present in patients who lack these features, however, testing should also be considered for patients with biallelic mutations in germline predisposition genes. The interpretation of mutations in patients with MDS is complex and must be performed with clinical context in mind. Ideally, clinician scientists, hematopathologists, expert guidelines, and entities performing these tests will help annotate and interpret the results to aid the ordering physician in applying them in practice and thus improving the care of patients with MDS.

\section{References}

1. Papaemmanuil E, Gerstung $M$, Malcovati L, et al. Clinical and biological implications of driver mutations in myelodysplastic syndromes. Blood 2013;122:3616-3627.

2. Haferlach T, Nagata Y, Grossmann V, et al. Landscape of genetic lesions in 944 patients with myelodysplastic syndromes. Leukemia 2014;28:241-247.

3. Greenberg PL, Stone RM, Al Kali A, et al. NCCN Clinical Practice Guidelines in Oncology: Myelodysplastic Syndromes, Version 2.2017. Accessed December 20, 2016. To view the most recent version of these guidelines, visit NCCN.org.

4. Bejar R, Stevenson K, Abdel-Wahab O, et al. Clinical effect of point mutations in myelodysplastic syndromes. N Engl J Med 2011;364:2496-2506.

5. Bejar R, Papaemmanuil E, Haferlach T, et al. Somatic mutations in MDS patients are associated with clinical features and predict prognosis independent of the IPSS-R: analysis of combined datasets from the International Working Group for Prognosis in MDS-Molecular Committee. Blood 2015;126:907. 
6. Bejar R. Clinical and genetic predictors of prognosis in myelodysplastic syndromes. Haematologica 2014;99:956964.

7. Bejar R, Stevenson KE, Caughey B, et al. Somatic mutations predict poor outcome in patients with myelodysplastic syndrome after hematopoietic stem-cell transplantation. J Clin Oncol 2014;32:2691-2698.

8. Della Porta MG, Galli A, Bacigalupo A, et al. Clinical effects of driver somatic mutations on the outcomes of patients with myelodysplastic syndromes treated with allogeneic hematopoietic stem-cell transplantation [published online ahead of print September 6, 2016]. J Clin Oncol, pii:JCO673616.

9. Malcovati L, Karimi M, Papaemmanuil E, et al. SF3B1 mutation identifies a distinct subset of myelodysplastic syndrome with ring sideroblasts. Blood 2015;126:233-241.

10. Valent P, Bain BJ, Bennett JM, et al. Idiopathic cytopenia of undetermined significance (ICUS) and idiopathic dysplasia of uncertain significance (IDUS), and their distinction from low risk MDS. Leuk Res 2012;36:1-5.

11. Arber DA, Orazi A, Hasserjian R, et al. The 2016 revision to the World Health Organization classification of myeloid neoplasms and acute leukemia. Blood 2016;127:2391-2405.

12. Greenberg PL, Tuechler H, Schanz J, et al. Cytopenia levels for aiding establishment of the diagnosis of myelodysplastic syndromes. Blood 2016;128:2096-2097.

13. Jaiswal S, Fontanillas $P$, Flannick J, et al. Age-related clonal hematopoiesis associated with adverse outcomes. $N$ Engl J Med 2014;371:2488-2498.

14. Genovese G, Kahler AK, Handsaker RE, et al. Clonal hematopoiesis and blood-cancer risk inferred from blood DNA sequence. N Engl J Med 2014;371:2477-2487.

15. Steensma DP, Bejar R, Jaiswal S, et al. Clonal hematopoiesis of indeterminate potential and its distinction from myelodysplastic syndromes. Blood 2015;126:9-16.

16. Link DC, Walter MJ. 'CHIP’ping away at clonal hematopoiesis. Leukemia 2016;30:1633-1635.

17. Kwok B, Hall JM, Witte JS, et al. MDS-associated somatic mutations and clonal hematopoiesis are common in idiopathic cytopenias of undetermined significance. Blood 2015;126:2355-2361.

18. Cargo CA, Rowbotham N, Evans PA, et al. Targeted sequencing identifies patients with preclinical MDS at high risk of disease progression. Blood 2015;126:2362-2365.

19. Fernandez-Pol S, Ma L, Ohgami RS, Arber DA. Significance of myelodysplastic syndrome-associated somatic variants in the evaluation of patients with pancytopenia and idiopathic cytopenias of undetermined significance. Mod Pathol 2016;29:996-1003.

20. Lewinsohn M, Brown AL, Weinel LM, et al. Novel germ line DDX41 mutations define families with a lower age of MDS/AML onset and lymphoid malignancies. Blood 2016;127:1017-1023.

21. Polprasert C, Schulze I, Sekeres MA, et al. Inherited and somatic defects in DDX41 in myeloid neoplasms. Cancer Cell 2015;27:658-670.

22. Babushok DV, Bessler M, Olson TS. Genetic predisposition to myelodysplastic syndrome and acute myeloid leukemia in children and young adults. Leuk Lymphoma 2016;57:520-536.

23. Nickels EM, Soodalter J, Churpek JE, Godley LA. Recognizing familial myeloid leukemia in adults. Ther Adv Hematol 2013;4:254-269.

24. Churpek JE, Marquez R, Neistadt B, et al. Inherited mutations in cancer susceptibility genes are common among survivors of breast cancer who develop therapy-related leukemia. Cancer 2016;122:304-311. 